St at i st i cal angul ar cor rel at i on coef $\mathrm{fici}$ ent $\mathrm{s}$ and second el ectron- pai $r$ moments for at ons

\begin{tabular}{|c|c|}
\hline 著者 & KOGA Toshi kat su \\
\hline $\begin{array}{l}\text { j our nal or } \\
\text { publ i cat } \mathrm{i} \text { on } \mathrm{title}\end{array}$ & The j our nal of chemi cal physi cs \\
\hline vol une & 115 \\
\hline number & 15 \\
\hline page $r$ ange & 6847- 6852 \\
\hline year & $2001-10-15$ \\
\hline URL & ht t p: //hdl . handl e. net /10258/799 \\
\hline
\end{tabular}




\title{
Statistical angular correlation coefficients and second electron-pair moments for atoms
}

\author{
Toshikatsu Koga \\ Department of Applied Chemistry, Muroran Institute of Technology, Muroran, Hokkaido 050-8585, Japan
}

(Received 1 May 2001; accepted 1 August 2001)

\begin{abstract}
Statistical correlation coefficients $\tau[f]$ introduced by Kutzelnigg et al. [Phys. Rev. 172, 49 (1968)] provide overall measures of the difference between the electron-pair density and the product of single-electron densities, where $f=f(\mathbf{r})$ is a probe function. It is shown that the angular correlation coefficient $\tau[\mathbf{r}]$, a particular case of $\tau[f]$ for $f(\mathbf{r})=\mathbf{r}$, is simply expressible in terms of the generalized electron-pair moments $\left\langle q^{2}\right\rangle_{(a, b)}$ with two real-valued parameters $a$ and $b$. Especially, the relation for $a=b=1$ means that $\tau[\mathbf{r}]$ is nothing but the difference between the center-of-mass motion and relative motion contributions in the second electron-pair moments. Conversely, the electron-pair moments $\left\langle q^{2}\right\rangle_{(a, b)}$ are obtained from the single-electron moment $\left\langle\Sigma_{i} r_{i}^{2}\right\rangle$ and the correlation coefficient $\tau[\mathbf{r}]$. The same is also true in momentum space. (C) 2001 American Institute of Physics. [DOI: 10.1063/1.1405010]
\end{abstract}

\section{INTRODUCTION}

In quantum chemistry, the electron correlation usually means (see, e.g., Ref. 1) the difference in the electron motion between the exact and Hartree-Fock solutions. However, there is another definition for the term correlation. When the concepts from mathematical statistics and probability theory are applied, the electron correlation means ${ }^{2}$ the manner in which the electron-pair density differs from the product of single-electron densities, and is described by the pair correlation density ${ }^{2}$ with six variables. In order to have simple numerical indices which provide overall measures for the statistical correlation of electrons, Kutzelnigg et al. ${ }^{3}$ introduced statistical correlation coefficients $\tau[f]$ defined by

$$
\tau[f] \equiv \frac{2 N\left\langle\sum_{i<j} f\left(\mathbf{r}_{i}\right) f\left(\mathbf{r}_{j}\right)\right\rangle-(N-1)\left\langle\sum_{i} f\left(\mathbf{r}_{i}\right)\right\rangle^{2}}{(N-1)\left(N\left\langle\sum_{i} f^{2}\left(\mathbf{r}_{i}\right)\right\rangle-\left\langle\sum_{i} f\left(\mathbf{r}_{i}\right)\right\rangle^{2}\right)^{2}},
$$

where $f(\mathbf{r})$ is a probe function and the angular brackets \langle\rangle stand for the expectation value over the $N$-electron $(N \geqslant 2)$ wave function $\Psi\left(\mathbf{x}_{1}, \ldots, \mathbf{x}_{N}\right)$ with $\mathbf{x}_{i} \equiv\left(\mathbf{r}_{i}, \sigma_{i}\right)$ being the combined position-spin coordinates of the electron $i$. The statistical correlation coefficients are bound ${ }^{3}$ as $-1 \leqslant \tau[f] \leqslant+1$, and electrons are perfectly correlated if $\tau[f]= \pm 1$, while they are independent or uncorrelated if $\tau[f]=0$. In a particular case of $f(\mathbf{r})=\mathbf{r}$, the correlation coefficient $\tau[f]$ for atoms simplifies to

$$
\tau[\mathbf{r}]=2\left\langle\sum_{i<j} \mathbf{r}_{i} \cdot \mathbf{r}_{j}\right\rangle /\left[(N-1)\left\langle\sum_{i} r_{i}^{2}\right\rangle\right],
$$

and $\tau[\mathbf{r}]$ is called the angular correlation coefficient, because $\mathbf{r}_{i} \cdot \mathbf{r}_{j}=r_{i} r_{j} \cos \theta_{i j}$, where $r_{i}=\left|\mathbf{r}_{i}\right|$ and $\theta_{i j}$ is the angle spanned by the two position vectors. Statistical correlation coefficients $\tau[\bar{f}]$ in momentum space are also defined by replacing $f(\mathbf{r})$ with $\bar{f}(\mathbf{p})$ in Eq. (1) and by considering the expectation value over the momentum wave function
$\Phi\left(\mathbf{y}_{1}, \ldots, \mathbf{y}_{N}\right)$, where $\mathbf{y}_{i} \equiv\left(\mathbf{p}_{i}, \sigma_{i}\right)$ is the combined momentum-spin coordinates of the electron $i$. Analogous to $\tau[f]$, the momentum-space correlation coefficient $\tau[\bar{f}]$ is bound by \pm 1 , and its particular case for $\bar{f}(\mathbf{p})=\mathbf{p}$ gives the momentum angular correlation coefficient,

$$
\tau[\mathbf{p}]=2\left\langle\sum_{i<j} \mathbf{p}_{i} \cdot \mathbf{p}_{j}\right\rangle /\left[(N-1)\left\langle\sum_{i} p_{i}^{2}\right\rangle\right] .
$$

Some numerical results of $\tau[f]$ were given in Ref. 3 for simple atoms and molecules. For helium and its isoelectronic analogs, the correlation coefficients were studied ${ }^{4-6}$ in detail not only in their ground states but also in many excited states. The Be-like ions were examined in Ref. 7. Thakkar pointed out ${ }^{8}$ that the angular correlation coefficient $\tau[\mathbf{r}]$ is connected with the form factor, the incoherent scattering function, and the minus first moment of the dipole oscillator strength density, and calculated $\tau[\mathbf{r}]$ for the first-row atoms. The $\tau[\mathbf{r}]$ values for atoms $\mathrm{He}$ through $\mathrm{Si}$ were given by Pathak $^{9}$ within the Hohenberg-Kohn-Sham density functional formalism. The statistical correlation coefficients were also examined in momentum space for He-like atoms, ${ }^{10,11}$ Li-like atoms, ${ }^{12}$ and $\mathrm{HeH}^{+}$molecule. ${ }^{13}$ Using the exactly soluble "harmonium" atom, King and Rothstein discussed ${ }^{14}$ that the correlation measured in the statistical sense does not necessarily measure the electron correlation in the energetic sense.

In the present paper, we show that the angular correlation coefficient $\tau[\mathbf{r}]$ can be simply expressed by means of the second moments $\left\langle q^{2}\right\rangle_{(a, b)}$ of the generalized electronpair density $g(q ; a, b)$. A special case of $a=b=1$ implies that $\tau[\mathbf{r}]$ is nothing but the difference between the center-ofmass motion (extracule) and relative motion (intracule) contributions measured by the second electron-pair moments. Conversely, the second electron-pair moments $\left\langle q^{2}\right\rangle_{(a, b)}$ are obtained from the single-electron moment $\left\langle\Sigma_{i} r_{i}^{2}\right\rangle$ and the angular correlation coefficient $\tau[\mathbf{r}]$. We find that the moment $\left\langle q^{2}\right\rangle_{(1, b)}$ takes the minimum at $b=-\tau[\mathbf{r}]$, and for statisti- 
cally uncorrelated wave functions the single-electron moment $\left\langle\Sigma_{i} r_{i}^{2}\right\rangle$ multiplied by $(N-1) / 2$ is the minimum of the electron-pair moments $\left\langle q^{2}\right\rangle_{(1, b)}$. Hartree atomic units are used throughout.

\section{ANGULAR CORRELATION COEFFICIENTS IN TERMS OF SECOND ELECTRON-PAIR MOMENTS}

The generalized electron-pair density function $g(q ; a, b)$ is defined ${ }^{15,16}$ by

$$
g(q ; a, b) \equiv\left(4 \pi q^{2}\right)^{-1}\left\langle\sum_{i<j} \delta\left(q-\left|a \mathbf{r}_{i}+b \mathbf{r}_{j}\right|\right)\right\rangle,
$$

where $a$ and $b$ are real-valued parameters and $\delta(x)$ is the one-dimensional Dirac delta function. The generalized electron-pair density $g(q ; a, b)$ represents the probability density function for the magnitude $\left|a \mathbf{r}_{i}+b \mathbf{r}_{j}\right|$ of the twoelectron vector $a \mathbf{r}_{i}+b \mathbf{r}_{j}$ of any pair of electrons $i$ and $j$ to be $q$, and is normalized to $N(N-1) / 2$, the number of electron pairs. The function $g(q ; a, b)$ with $a=1$ is of our particular interest, since $g(q ; 1, b)$ smoothly connects ${ }^{16}$ the singleelectron density $\rho(r)$, the electron-pair intracule $h(u)$, and extracule $d(R)$ densities ${ }^{17-21}$ as a function of the parameter $b$, which were originally defined in a completely independent manner. Namely, $\quad g(q ; 1,-1)=h(q), \quad g(q ; 1,0)=[(N$ $-1) / 2] \rho(q)$, and $g(q ; 1,+1)=d(q / 2) / 8$, where the last equality follows from a scaling relation ${ }^{15}$ of $g(q ; a, b)$.

If we introduce the moments $\left\langle q^{k}\right\rangle_{(a, b)}$ of the density $g(q ; a, b)$,

$$
\left\langle q^{k}\right\rangle_{(a, b)} \equiv 4 \pi \int_{0}^{\infty} d q q^{k+2} g(q ; a, b)=\left\langle\sum_{i<j}\left|a \mathbf{r}_{i}+b \mathbf{r}_{j}\right|^{k}\right\rangle,
$$

then the second moments $\left\langle q^{2}\right\rangle_{(a, b)}$ are known ${ }^{22,23}$ to satisfy the sum rules

$$
\begin{aligned}
& \left\langle q^{2}\right\rangle_{(a, b)}+\left\langle q^{2}\right\rangle_{(a,-b)}=\left(a^{2}+b^{2}\right)(N-1)\left\langle\sum_{i} r_{i}^{2}\right\rangle, \\
& \left\langle q^{2}\right\rangle_{(a, b)}-\left\langle q^{2}\right\rangle_{(a,-b)}=4 a b\left\langle\sum_{i<j} \mathbf{r}_{i} \cdot \mathbf{r}_{j}\right\rangle .
\end{aligned}
$$

Therefore, the angular correlation coefficient $\tau[\mathbf{r}]$, given by Eq. (2), is rewritten in general as

$$
\tau[\mathbf{r}]=\frac{\left(a^{\prime 2}+b^{\prime 2}\right)\left(\left\langle q^{2}\right\rangle_{(a, b)}-\left\langle q^{2}\right\rangle_{(a,-b)}\right)}{2 a b\left(\left\langle q^{2}\right\rangle_{\left(a^{\prime}, b^{\prime}\right)}+\left\langle q^{2}\right\rangle_{\left(a^{\prime},-b^{\prime}\right)}\right)},
$$

where $a b \neq 0$ but it may happen that $a^{\prime}=a$ and/or $b^{\prime}=b$. Apart from the constant $\left(a^{\prime 2}+b^{\prime 2}\right) /(2 a b)$, the correlation coefficient $\tau[\mathbf{r}]$ is the ratio between the difference and the sum of two sets of the second electron-pair moments, which are symmetric with respect to the second parameter $b$ or $b^{\prime}$. For a special case of $a^{\prime}=a$ and $b^{\prime}=0$, Eq. (7a) reduces to

$$
\tau[\mathbf{r}]=\frac{a\left(\left\langle q^{2}\right\rangle_{(a, b)}-\left\langle q^{2}\right\rangle_{(a,-b)}\right)}{4 b\left\langle q^{2}\right\rangle_{(a, 0)}} .
$$

Since the right-hand side of Eq. (7b) is the product of $a /\left(2\left\langle q^{2}\right\rangle_{(a, 0)}\right)$ and $\left(\left\langle q^{2}\right\rangle_{(a, b)}-\left\langle q^{2}\right\rangle_{(a,-b)}\right) /(2 b)$, Eq. (7b) means that for a given value of $a, \tau[\mathbf{r}]$ is proportional to the slope of the moment $\left\langle q^{2}\right\rangle_{(a, b)}$ as a function of the parameter $b$. If we consider the zero- $b$ limit, Eq. (7b) is therefore rewritten as

$$
\tau[\mathbf{r}]=\frac{a}{2}\left[\frac{\partial}{\partial b} \ln \left\langle q^{2}\right\rangle_{(a, b)}\right]_{b=0} .
$$

For $a^{\prime}=1, b^{\prime}=0$, and $a b \neq 0$, Eq. (7a) yields

$$
\tau[\mathbf{r}]=\frac{1}{2 a b}\left\{\frac{\left\langle q^{2}\right\rangle_{(a, b)}}{\left\langle q^{2}\right\rangle_{(1,0)}}-\left(a^{2}+b^{2}\right)\right\} .
$$

Another special case of Eq. (7a) for $a=a^{\prime}=b=b^{\prime}=1$ reads

$$
\tau[\mathbf{r}]=\frac{4\left\langle R^{2}\right\rangle-\left\langle u^{2}\right\rangle}{4\left\langle R^{2}\right\rangle+\left\langle u^{2}\right\rangle}
$$

since $^{15}\left\langle q^{k}\right\rangle_{(1,-1)}=\left\langle u^{k}\right\rangle$ and $\left\langle q^{k}\right\rangle_{(1,+1)}=2^{k}\left\langle R^{k}\right\rangle$, where $\left\langle u^{k}\right\rangle$ and $\left\langle R^{k}\right\rangle$ are the intracule and extracule moments ${ }^{17-21}$ associated with the electron-pair densities $h(u)$ and $d(R)$, respectively. Equation (7e) shows that $\tau[\mathbf{r}]$ essentially measures the difference between the center-of-mass and relative motions of electron pairs through the second moments.

When we introduce ${ }^{15,16}$ the generalized electron-pair density $\bar{g}(t ; a, b)$ and associated moments $\left\langle t^{k}\right\rangle_{(a, b)}$ in momentum space,

$$
\begin{aligned}
& \bar{g}(t ; a, b) \equiv\left(4 \pi t^{2}\right)^{-1}\left\langle\sum_{i<j} \delta\left(t-\left|a \mathbf{p}_{i}+b \mathbf{p}_{j}\right|\right)\right\rangle, \\
& \left\langle t^{k}\right\rangle_{(a, b)} \equiv 4 \pi \int_{0}^{\infty} d t t^{k+2} \bar{g}(t ; a, b)=\left\langle\sum_{i<j}\left|a \mathbf{p}_{i}+b \mathbf{p}_{j}\right|^{k}\right\rangle,
\end{aligned}
$$

exactly the same discussion as in position space results in several rigorous relations between $\tau[\mathbf{p}]$ and $\left\langle t^{2}\right\rangle_{(a, b)}$. For example, the relations corresponding to Eqs. (7a) and (7e) are

$$
\begin{aligned}
& \tau[\mathbf{p}]=\frac{\left(a^{\prime 2}+b^{\prime 2}\right)\left(\left\langle t^{2}\right\rangle_{(a, b)}-\left\langle t^{2}\right\rangle_{(a,-b)}\right)}{2 a b\left(\left\langle t^{2}\right\rangle_{\left(a^{\prime}, b^{\prime}\right)}+\left\langle t^{2}\right\rangle_{\left(a^{\prime},-b^{\prime}\right)}\right)}, \\
& \tau[\mathbf{p}]=\frac{4\left\langle P^{2}\right\rangle-\left\langle v^{2}\right\rangle}{4\left\langle P^{2}\right\rangle+\left\langle v^{2}\right\rangle},
\end{aligned}
$$

where $\left\langle v^{k}\right\rangle$ and $\left\langle P^{k}\right\rangle$ are the moments ${ }^{18-21}$ associated with the electron-pair intracule $\bar{h}(v)$ and extracule $\bar{d}(P)$ densities in momentum space, respectively.

\section{SECOND ELECTRON-PAIR MOMENTS IN TERMS OF ANGULAR CORRELATION COEFFICIENTS}

Above we have shown that the angular correlation coefficient $\tau[\mathbf{r}]$ can be simply obtained from the second electronpair moments $\left\langle q^{2}\right\rangle_{(a, b)}$. Conversely, the moments $\left\langle q^{2}\right\rangle_{(a, b)}$ are expressible in terms of the single-electron moments $\left\langle\Sigma_{i} r_{i}^{2}\right\rangle$ and the correlation coefficient $\tau[\mathbf{r}]$. Combining Eqs. (2), (6a), and (6b), we have

$$
\begin{aligned}
\left\langle q^{2}\right\rangle_{(a, b)} & =\frac{1}{2}(N-1)\left\langle\sum_{i} r_{i}^{2}\right\rangle\left(a^{2}+b^{2}+2 a b \tau[\mathbf{r}]\right) \\
& =\left\langle q^{2}\right\rangle_{(1,0)}\left(a^{2}+b^{2}+2 a b \tau[\mathbf{r}]\right),
\end{aligned}
$$




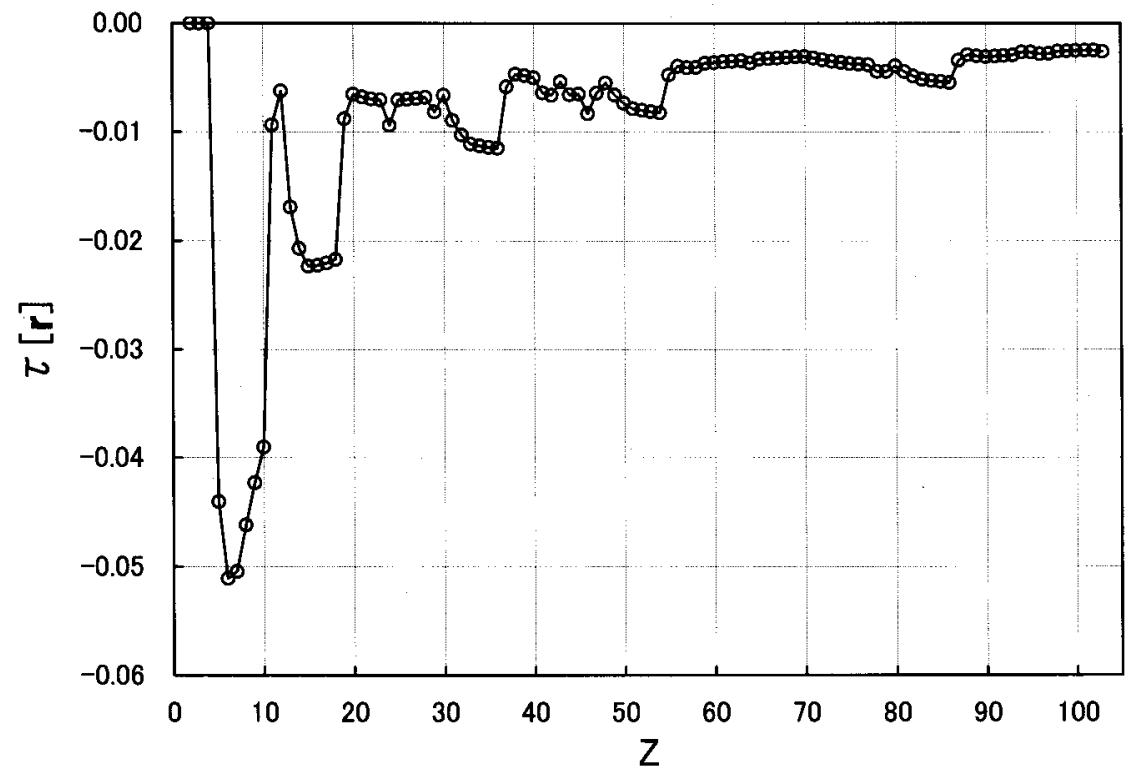

FIG. 1. The Hartree-Fock limit angular correlation coefficients $\tau[\mathbf{r}]$ in position space as a function of atomic number $Z$.

whose particular cases for $a=b=1$ (extracule moment) and $a=-b=1$ (intracule moment) are:

$$
\begin{aligned}
& \left\langle R^{2}\right\rangle=\frac{1}{4}(N-1)\left\langle\sum_{i} r_{i}^{2}\right\rangle(1+\tau[\mathbf{r}]), \\
& \left\langle u^{2}\right\rangle=(N-1)\left\langle\sum_{i} r_{i}^{2}\right\rangle(1-\tau[\mathbf{r}]) .
\end{aligned}
$$

Equations (11a)-(11c) are consistent with a general relation $^{23}$

$$
\left\langle q^{2}\right\rangle_{(a, b)}=(a+b)^{2}\left\langle R^{2}\right\rangle+\frac{1}{4}(a-b)^{2}\left\langle u^{2}\right\rangle .
$$

For $a=1$, Eq. (11a) is rearranged as

$$
\left\langle q^{2}\right\rangle_{(1, b)}=\frac{1}{2}(N-1)\left\langle\sum_{i} r_{i}^{2}\right\rangle\left\{(b+\tau[\mathbf{r}])^{2}+1-\tau^{2}[\mathbf{r}]\right\} .
$$

Thus the moment $\left\langle q^{2}\right\rangle_{(1, b)}$ is a quadratic function of $b$ and takes the minimum $\frac{1}{2}(N-1)\left\langle\sum_{i} r_{i}^{2}\right\rangle\left(1-\tau^{2}[\mathbf{r}]\right)$ at $b$ $=-\tau[\mathbf{r}]$. For uncorrelated wave functions, $\tau[\mathbf{r}]=0$ and hence the single-electron moment $\left\langle\Sigma_{i} r_{i}^{2}\right\rangle$, multiplied by $(N$ $-1) / 2$, is the minimum of the electron-pair moment $\left\langle q^{2}\right\rangle_{(1, b)}$ as a function of the parameter $b$. If we consider the region $-1 \leqslant b \leqslant+1$, Eq. (13) says that the maximum of $\left\langle q^{2}\right\rangle_{(1, b)}$ occurs for the intracule moment $\left\langle u^{2}\right\rangle=\left\langle q^{2}\right\rangle_{(1,-1)}$ when $\tau[\mathbf{r}]<0$ while for the extracule moment $4\left\langle R^{2}\right\rangle=\left\langle q^{2}\right\rangle_{(1,+1)}$ when $\tau[\mathbf{r}]>0$. From Eqs. (11b) and (11c), we also find

$$
4\left\langle R^{2}\right\rangle-\left\langle u^{2}\right\rangle=2(N-1)\left\langle\sum_{i} r_{i}^{2}\right\rangle \tau[\mathbf{r}],
$$

which implies that the equality in an approximate isomorphism, ${ }^{15}\left\langle u^{2}\right\rangle \cong 4\left\langle R^{2}\right\rangle$, occurs only when $\tau[\mathbf{r}]=0$.

Based on Eq. (6a) with $a=b=1$, it has been pointed out in the literature ${ }^{22}$ that the single-electron moment $\left\langle\Sigma_{i} r_{i}^{2}\right\rangle$ can be precisely decomposed into the contributions from the relative (rel) and center-of-mass (com) motions.

$$
\begin{aligned}
& \left\langle\sum_{i} r_{i}^{2}\right\rangle=\left\langle\sum_{i} r_{i}^{2}\right\rangle_{\mathrm{rel}}+\left\langle\sum_{i} r_{i}^{2}\right\rangle_{\mathrm{com}}, \\
& \left\langle\sum_{i} r_{i}^{2}\right\rangle_{\mathrm{rel}}=\frac{\left\langle u^{2}\right\rangle}{2(N-1)},\left\langle\sum_{i} r_{i}^{2}\right\rangle_{\mathrm{com}}=\frac{2\left\langle R^{2}\right\rangle}{N-1} .
\end{aligned}
$$

Then the ratio of the two contributions is

$$
\begin{aligned}
\left\langle\sum_{i} r_{i}^{2}\right\rangle_{\text {rel }} /\left\langle\sum_{i} r_{i}^{2}\right\rangle_{\text {com }} & =\left\langle u^{2}\right\rangle /\left(4\left\langle R^{2}\right\rangle\right) \\
& =(1-\tau[\mathbf{r}]) /(1+\tau[\mathbf{r}]),
\end{aligned}
$$

which clarifies that the relative motion contribution is larger if $\tau[\mathbf{r}]<0$, whereas the center-of-mass motion contribution is if $\tau[\mathbf{r}]>0$.

The corresponding expressions for $\left\langle t^{2}\right\rangle_{(a, b)},\left\langle v^{2}\right\rangle,\left\langle P^{2}\right\rangle$, and related quantities, in terms of $\left\langle\Sigma_{i} p_{i}^{2}\right\rangle$ and $\tau[\mathbf{p}]$, are immediately obtained from the above results in position space, if the names of variables are appropriately replaced with their momentum-space counterparts. The mathematical structure remains unaltered, and the same discussion as in position space holds in momentum space as well.

\section{NUMERICAL RESULTS}

In position space, systematic data of the electron-pair intracule $\left\langle u^{2}\right\rangle$ and extracule $\left\langle R^{2}\right\rangle$ moments were reported in Refs. $24-26$ at the Hartree-Fock limit level for the 102 atoms from $\mathrm{He}$ (atomic number $Z=2)$ to $\operatorname{Lr}(Z=103)$ in their experimental ground states. ${ }^{27,28}$ Using these data, we have calculated the angular correlation coefficients $\tau[\mathbf{r}]$ based on Eq. (7e). The results are plotted in Fig. 1 as a function of $Z$. For the first three atoms $\mathrm{He}, \mathrm{Li}$, and $\mathrm{Be}$, where only $s$ orbitals are occupied, $\tau[\mathbf{r}]$ is zero, and the electrons are statistically uncorrelated when measured by the probe function $f(\mathbf{r})=\mathbf{r}$. For the remaining 99 atoms, the correlation coefficients are negative with no exceptions. These electrons are negatively 
TABLE I. Comparison of the angular correlation coefficients $\tau[\mathbf{r}]$ in position space.

\begin{tabular}{lcccc}
\hline \hline & & & \multicolumn{2}{c}{$\begin{array}{c}\text { Calculated from } \\
\text { electron-pair moments }\end{array}$} \\
\cline { 3 - 5 } Atom & $\begin{array}{c}\text { Density- } \\
\text { functional }^{\mathrm{a}}\end{array}$ & $\begin{array}{c}\text { Configuration } \\
\text { interaction }^{\mathrm{b}}\end{array}$ & $\begin{array}{c}\text { Hartree-Fock } \\
\text { limit }^{\mathrm{c}}\end{array}$ & $\begin{array}{c}\text { Explicitly } \\
\text { correlated }^{\mathrm{d}}\end{array}$ \\
\hline $\mathrm{He}$ & -0.04087 & $-0.05414^{\mathrm{e}}$ & 0 & -0.054242 \\
$\mathrm{Li}$ & -0.03329 & -0.0032 & 0 & -0.004089 \\
$\mathrm{Be}$ & -0.06179 & -0.0975 & 0 & -0.080083 \\
$\mathrm{~B}$ & -0.09930 & -0.0816 & -0.044043 & -0.070145 \\
$\mathrm{C}$ & -0.05350 & -0.0688 & -0.051095 & -0.065773 \\
$\mathrm{~N}$ & -0.05400 & -0.0569 & -0.050444 & -0.056535 \\
$\mathrm{O}$ & -0.05053 & -0.0576 & -0.046171 & -0.051658 \\
$\mathrm{~F}$ & -0.04655 & -0.0479 & -0.042321 & -0.046520 \\
$\mathrm{Ne}$ & -0.04387 & -0.0463 & -0.039030 & -0.044461 \\
\hline \hline
\end{tabular}

${ }^{\mathrm{a}}$ Hohenberg-Kohn-Sham method with Bartolotti exchange-correlation functional. Reference 9.

${ }^{\mathrm{b}}$ Reference 8 except He.

${ }^{\mathrm{c} C a l c u l a t e d}$ from the Hartree-Fock limit $\left\langle u^{2}\right\rangle$ and $\left\langle R^{2}\right\rangle$ values in Refs. 24, 25.

${ }^{\mathrm{d}}$ Calculated from the explicitly correlated $\left\langle u^{2}\right\rangle$ and $\left\langle R^{2}\right\rangle$ values in Refs. 30-35.

${ }^{\mathrm{e}}$ Calculated from the data in Ref. 36.

correlated or the angle $\theta_{i j}$ spanned by two position vectors $\mathbf{r}_{i}$ and $\mathbf{r}_{j}$ is greater than $\pi / 2$ in an average sense. The values of $\tau[\mathbf{r}]$ in Fig. 1 show a periodic behavior reflecting the valence electronic configuration: Within a period, the values are generally larger when the valence $p$ subshell is occupied. The difference in the valence $s^{0}, s^{1}$, and $s^{2}$ configurations is clearly observed for the first $(Z=21-30)$ and second $(Z$ $=39-48)$ transition atoms. The magnitude of the negative correlation decreases in general as $Z$ increases. This is due to the fact that the denominator $4\left\langle R^{2}\right\rangle+\left\langle u^{2}\right\rangle$ in Eq. (7e) steadily increases with the increasing number of electron pairs, while some electron pairs give ${ }^{29}$ zero contribution to the numerator $4\left\langle R^{2}\right\rangle-\left\langle u^{2}\right\rangle$. The result can also be interpreted as that the increase in nuclear charge contributes to make electrons more hydrogenic or independent. Within the Hartree-Fock approximation, the largest negative correlation is observed for the carbon atom, where $\tau[\mathbf{r}]=-0.051$.

For the nine atoms $\mathrm{He}$ through $\mathrm{Ne}$, the moments $\left\langle u^{2}\right\rangle$ and $\left\langle R^{2}\right\rangle$ from explicitly correlated ( $r_{i j}$-terms in wave functions) calculations are also available in the literature. ${ }^{30-35}$ In Table I, the angular correlation coefficients $\tau[\mathbf{r}]$ from the explicitly correlated wave functions are compared with those from the Hartree-Fock limit wave functions and with those from density-functional ${ }^{8}$ and configuration interaction ${ }^{9,36}$ calculations. When we go beyond the Hartree-Fock approximation, all the atoms, including $\mathrm{He}-\mathrm{Be}$, have negative correlation coefficients and the so-called electron correlation effect works to increase the magnitudes of negative angular correlations. Within the second period atoms, the magnitude $|\tau[\mathbf{r}]|$ from the explicitly correlated and configuration interaction calculations is largest for the Be atom, and monotonically (except the $\mathrm{O}$ atom in the configuration interaction results) decreases toward the $\mathrm{Ne}$ atom. However, the densityfunctional calculations predict the greatest negative correlation for the B atom. The negative angular correlation of electrons is exceptionally small in the $\mathrm{Li}$ atom with a singly occupied valence $s$ shell.

In all the calculations, the values of $\tau[\mathbf{r}]$ in position space are negative or zero. According to Eq. (16), this im- plies that the relative motion contribution is never smaller than the center-of-mass motion contribution, when the single-electron moment $\left\langle\Sigma_{i} r_{i}^{2}\right\rangle$ is partitioned.

In momentum space, the Hartree-Fock limit values of the second intracule $\left\langle v^{2}\right\rangle$ and extracule $\left\langle P^{2}\right\rangle$ moments were reported in Refs. 24, 25, 37 for the 102 atoms from He to Lr. The momentum angular correlation coefficients $\tau[\mathbf{p}]$ have been calculated based on Eq. (10b) and are plotted in Fig. 2 as a function of $Z$. Again we find that the correlation coefficients are zero for the first three atoms, and are negative for the remaining 99 atoms. The $Z$ dependence of $\tau[\mathbf{p}]$ shows a simple structure with a single minimum at the $\mathrm{Ne}$ atom, where the negative correlation is largest $(\tau[\mathbf{p}]=-0.013)$ in momentum space. The result suggests that the momentumspace angular correlation coefficient predominantly reflects the statistical correlation of inner electrons. Among inner electron pairs, the $1 s 2 p$ and $2 s 2 p$ pairs are considered to be the major origin, since the orbital pairs with the same inversion symmetry give ${ }^{29}$ vanishing contributions to $4\left\langle P^{2}\right\rangle$ $-\left\langle v^{2}\right\rangle$. Therefore, the $\tau[\mathbf{p}]$ value decreases until the $2 p$ subshell is fully occupied at the $\mathrm{Ne}$ atom, and after that it gradually increases for the same reason as in position space.

The momentum-space angular correlation coefficients $\tau[\mathbf{p}]$ have also been calculated from the explicitly correlated values $^{34,38}$ of the electron-pair moments $\left\langle v^{2}\right\rangle$ and $\left\langle P^{2}\right\rangle$ for the atoms He through Ne. Table II summarizes the results and compares them with the corresponding Hartree-Fock limit and configuration interaction values. In the table, the electron correlation effect is found to increase the $\tau[\mathbf{p}]$ values for all the nine atoms. In fact, $\tau[\mathbf{p}]$ is positive for the four atoms $\mathrm{He}-\mathrm{B}$. In these atoms, the average angle $\bar{\theta}_{i j}$ between two momentum vectors $\mathbf{p}_{i}$ and $\mathbf{p}_{j}$ is less than $\pi / 2$, and the momenta of two electrons tend to be directed to the same direction. Krause et al. ${ }^{11}$ proposed for the He atom a classical picture of bending and antisymmetric stretching motions of two electrons for the positive value of $\tau[\mathbf{p}]$. However, $\tau[\mathbf{p}]$ changes to a negative value for the atoms $\mathrm{C}-\mathrm{Ne}$ and the momenta of two electrons tend to be directed to the opposite directions, as we observed in position space. At present, we 


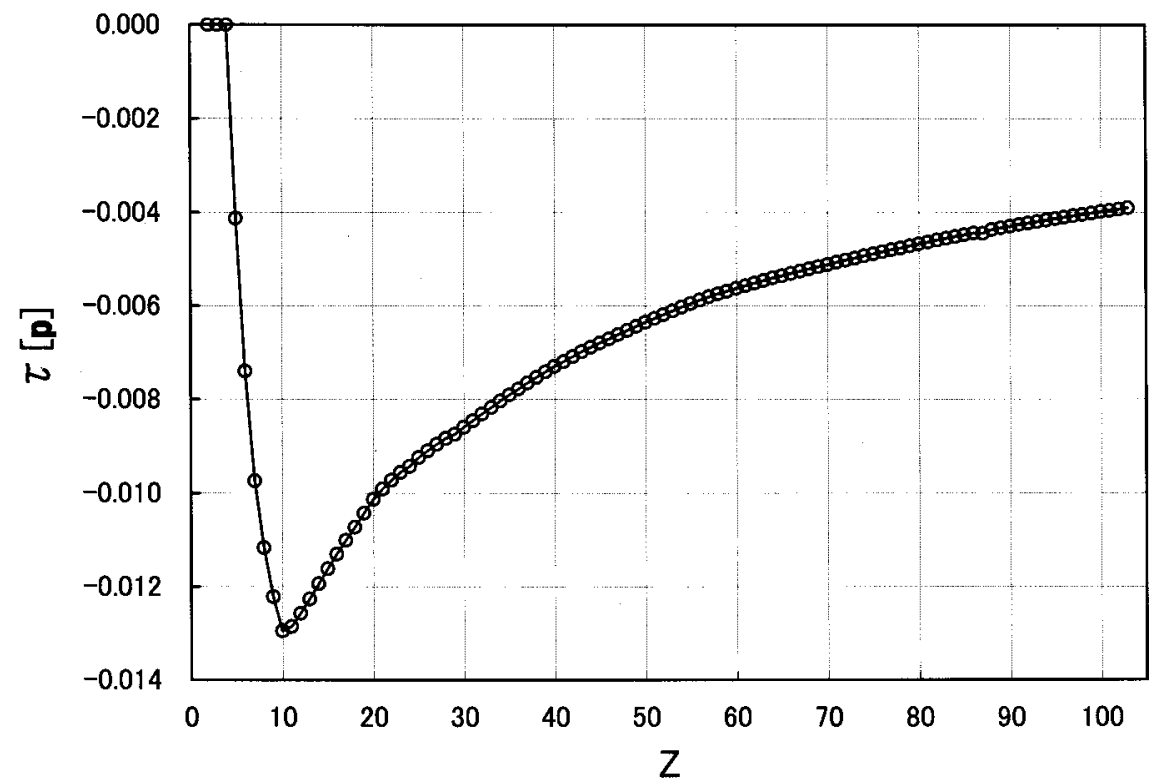

FIG. 2. The Hartree-Fock limit angular correlation coefficients $\tau[\mathbf{p}]$ in momentum space as a function of atomic number $Z$.

do not have any answer to the question why the sign of $\tau[\mathbf{p}]$ switches between the $\mathrm{B}$ and $\mathrm{C}$ atoms, and we have to await further investigations. Table II also shows that in momentum space, the inclusion of electron correlation effects reduces the magnitude of the statistical correlation of electrons for atoms $\mathrm{C}-\mathrm{Ne}$. Clearly, the results are not parallel to those obtained in position space and suggest that the problem of the electron correlations should be studied in both spaces.

Finally, we note that the magnitudes of $\tau[\mathbf{r}]$ and $\tau[\mathbf{p}]$ are small and never exceed 0.1 in all the cases examined. Since Eq. (13) says that $\left\langle q^{2}\right\rangle_{(1, b)}$ takes the minimum at $b$ $=-\tau[\mathbf{r}]$, a single-electron property $[(N-1) / 2]\left\langle\sum_{i} r_{i}^{2}\right\rangle$ $=\left\langle q^{2}\right\rangle_{(1,0)}$ is very close to the minimum value of the generalized electron-pair moment $\left\langle q^{2}\right\rangle_{(1, b)}$ as a function of $b$. The same discussion holds for the corresponding momentumspace properties $\left\langle t^{2}\right\rangle_{(1, b)}$ and $[(N-1) / 2]\left\langle\Sigma_{i} p_{i}^{2}\right\rangle=\left\langle t^{2}\right\rangle_{(1,0)}$.

TABLE II. Comparison of the angular correlation coefficients $\tau[\mathbf{p}]$ in momentum space.

\begin{tabular}{lccc}
\hline \hline & \multicolumn{2}{c}{$\begin{array}{c}\text { Calculated from } \\
\text { electron-pair moments }\end{array}$} \\
\cline { 3 - 4 } Atom & $\begin{array}{c}\text { Configuration } \\
\text { interaction }^{2}\end{array}$ & $\begin{array}{c}\text { Hartree-Fock } \\
\text { limit }^{\mathrm{a}}\end{array}$ & $\begin{array}{c}\text { Explicitly } \\
\text { correlated }^{\mathrm{b}}\end{array}$ \\
\hline $\mathrm{He}$ & $+0.055931^{\mathrm{c}}$ & 0 & +0.054569 \\
$\mathrm{Li}$ & $+0.022020^{\mathrm{d}}$ & 0 & +0.018197 \\
$\mathrm{Be}$ & 0 & +0.009744 \\
$\mathrm{~B}$ & & -0.004126 & +0.004641 \\
$\mathrm{C}$ & -0.007398 & -0.002141 \\
$\mathrm{~N}$ & -0.009740 & -0.006559 \\
$\mathrm{O}$ & -0.011170 & -0.007131 \\
$\mathrm{~F}$ & -0.012212 & -0.008483 \\
$\mathrm{Ne}$ & -0.012946 & -0.010135 \\
\hline \hline
\end{tabular}

${ }^{\mathrm{a} C a l c u l a t e d}$ from the Hartree-Fock limit $\left\langle v^{2}\right\rangle$ and $\left\langle P^{2}\right\rangle$ values in Refs. 24, 25.

${ }^{\mathrm{b}}$ Calculated from the explicitly correlated $\left\langle v^{2}\right\rangle$ and $\left\langle P^{2}\right\rangle$ values in Refs. 34, 37.

${ }^{\mathrm{c}}$ Calculated from the data in Ref. 13.

${ }^{\mathrm{d}}$ Calculated from the data in Ref. 12.

\section{SUMMARY}

We have shown that the angular correlation coefficient $\tau[\mathbf{r}]$ is simply expressed in terms of the generalized electronpair moments $\left\langle q^{2}\right\rangle_{(a, b)}$ with two real-valued parameters $a$ and $b$. In particular, the relation for $a=b=1$ means that $\tau[\mathbf{r}]$ measures the difference between the center-of-mass motion and relative motion contributions of electrons by means of the second electron-pair moments. Conversely, the electronpair moments $\left\langle q^{2}\right\rangle_{(a, b)}$ are obtained from the single-electron moment $\left\langle\Sigma_{i} r_{i}^{2}\right\rangle$ and the correlation coefficient $\tau[\mathbf{r}]$. The same is also true in momentum space. Numerical results of the angular correlation coefficients $\tau[\mathbf{r}]$ in position space and $\tau[\mathbf{p}]$ in momentum space have been presented and discussed for neutral atoms in their ground states.

\section{ACKNOWLEDGMENT}

This work was supported in part by a Grant-in-Aid for Scientific Research from the Ministry of Education of Japan.

${ }^{1}$ A. C. Hurley, Electron Correlation in Small Molecules (Academic, London, 1976).

${ }^{2}$ R. McWeeny, Rev. Mod. Phys. 32, 335 (1960).

${ }^{3}$ W. Kutzelnigg, G. Del Re, and G. Berthier, Phys. Rev. 172, 49 (1968).

${ }^{4}$ K. E. Banyard and D. J. Ellis, J. Phys. B 8, 2311 (1975).

${ }^{5}$ A. J. Thakkar and V. H. Smith, Jr., Phys. Rev. A 23, 473 (1981).

${ }^{6}$ N. M. Cann, R. J. Boyd, and A. J. Thakkar, Int. J. Quantum Chem., Symp. 27, 33 (1993).

${ }^{7}$ K. E. Banyard and M. M. Mashat, J. Chem. Phys. 67, 1405 (1977).

${ }^{8}$ A. J. Thakkar, Phys. Rev. A 25, 1820 (1982).

${ }^{9}$ R. K. Pathak, Phys. Rev. A 31, 2806 (1985).

${ }^{10}$ K. E. Banyard and J. C. Moore, J. Phys. B 10, 2781 (1977).

${ }^{11}$ J. L. Krause, J. D. Morgan III, and R. S. Berry, Phys. Rev. A 35, 3189 (1987).

${ }^{12}$ K. E. Banyard, K. H. Al-Bayati, and P. K. Youngman, J. Phys. B 21, 3177 (1988).

${ }^{13}$ C. E. Reed and K. E. Banyard, J. Phys. B 13, 1519 (1980).

${ }^{14}$ F. W. King and S. M. Rothstein, Phys. Rev. A 21, 1376 (1980).

${ }^{15}$ T. Koga, Theor. Chem. Acc. 105, 96 (2000).

${ }^{16}$ T. Koga, J. Chem. Phys. 114, 72 (2001); ibid., (E) 114, 9194 (2001).

${ }^{17}$ A. J. Coleman, Int. J. Quantum Chem., Symp. 1, 457 (1967).

${ }^{18}$ A. J. Thakkar, in Density Matrices and Density Functionals, edited by R. 
M. Erdahl and V. H. Smith, Jr. (Reidel, Dordrecht, 1987), pp. 553-581.

${ }^{19}$ R. J. Boyd and J. M. Ugalde, in Computational Chemistry, Part A, edited by S. Fraga (Elsevier, Amsterdam, 1992), pp. 273-299.

${ }^{20}$ E. Valderrama, J. M. Ugalde, and R. J. Boyd, in Many-Electron Densities and Reduced Density Matrices, edited by J. Cioslowski (Kluwer Academic/Plenum, New York, 2000), pp. 231-248.

${ }^{21}$ T. Koga, in Many-Electron Densities and Reduced Density Matrices, edited by J. Cioslowski (Kluwer Academic/Plenum, New York, 2000), pp. 267-298.

${ }^{22}$ T. Koga, J. Chem. Phys. 114, 2511 (2001).

${ }^{23}$ T. Koga and H. Matsuyama, J. Chem. Phys. (in press).

${ }^{24}$ T. Koga and H. Matsuyama, J. Chem. Phys. 107, 8510 (1997).

${ }^{25}$ T. Koga and H. Matsuyama, J. Chem. Phys. 108, 3424 (1998).

${ }^{26}$ T. Koga and H. Matsuyama, J. Chem. Phys. 111, 9191 (1999).

${ }^{27}$ C. E. Moore, Ionization Potentials and Ionization Limits Derived from the
Analysis of Optical Spectra, NSRDS-NBS 34 (Natl. Bur. Stand. U.S., Washington, D.C., 1970).

${ }^{28}$ H. L. Anderson, A Physicist's Desk Reference (AIP, New York, 1989), p. 94.

${ }^{29}$ T. Koga, J. Chem. Phys. 114, 8382 (2001).

${ }^{30}$ C. L. Pekeris, Phys. Rev. 115, 1216 (1959).

${ }^{31}$ F. W. King, Phys. Rev. A 40, 1735 (1989).

${ }^{32}$ J. Komasa, W. Cencek, and J. Rychlewski, Phys. Rev. A 52, 4500 (1995).

${ }^{33}$ A. Sarsa, F. J. Galvez, and E. Buendia, J. Chem. Phys. 109, 7075 (1998).

${ }^{34}$ F. J. Galvez, E. Buendia, and A. Sarsa, J. Chem. Phys. 111, 3319 (1999).

${ }^{35}$ T. Koga and H. Matsuyama, Int. J. Quantum Chem. 74, 455 (1999).

${ }^{36}$ J. W. Cooper and J. B. Martin, Phys. Rev. 131, 1183 (1963).

${ }^{37}$ T. Koga and H. Matsuyama, J. Chem. Phys. 113, 10114 (2000).

${ }^{38}$ A. Sarsa, F. J. Galvez, and E. Buendia, J. Chem. Phys. 110, 5721 (1999). 